\title{
The Impact of Tianjin-Baoding Intercity Railway on the Traffic Pattern of the Jing-Jin-Ji Urban Agglomeration
}

\author{
QI Lei ${ }^{1, a^{*}}$, GUO Jing ${ }^{2}$ \\ ${ }^{1}$ Hebei Cansina Engineering Design Co.,Ltd, Baoding,Hebei,China,071000 \\ ${ }^{2}$ College of Agronomy,Agricultural University of Hebei, Baoding,Hebei,China,071000 \\ aEmail: aphelion.qi@hotmail.com
}

Keywords: Tianjin-Baoding Intercity Railway, Traffic Pattern, Jing-Jin-Ji Urban Agglomeration, accessibility, time-space compression

\begin{abstract}
The construction of transportation infrastructure has a profound influence on regional and urban development. The high-speed rail can greatly enhance the transport and economic connection of the urban agglomerations separated. This research aims to examine the impact of the Tianjin-Baoding Intercity Railway (TBIR) on the transportation pattern in the Jing-Jin-Ji urban agglomerations. With the help of ArcGIS Desktop10, the traditional accessibility assessment model was conducted to analyze the changes of accessibility before and after the construction of TBIR. The model was modified to illustrate the non-transportation-network grids in details. The grids were evaluated by geographical factors (water surface, elevation or slope) or land use types instead of being regarded as homogeneous in traditional model. The result shows that the time accessibility of Tianjin and Baoding is greatly improved with the TBIR, while the other cities in the urban agglomerations are not as significantly improved as the former two. The research believes that the TBIR exerts a significant influence on the transportation pattern of the Jing-Jin-Ji urban agglomerations, compresses the time and space between Tianjin and Baoding, and it significantly improves the importance of Baoding, which may change the urban agglomerations from "sin-Hub" to "bi-Hubs". Finally, this paper analyzes the impact of the TBIR on the industry of commerce and trade, tourism, real estate, regional logistics network and so on.
\end{abstract}

\section{Introduction}

Transportation network system plays a key role in the development of urban agglomeration and the formation of spatial structure, and the construction of new facilities has a far-reaching influence on the development of urban agglomeration. According to the space of flows view, inter city connectivity enhancement will weaken the physical proximity. Railway, especially the high-speed railway has a significant "time-space compression" benefit, will greatly reduce the time distance between cities, and thus the formation and change of the regional spatial structure. In this paper, the newly built opened across Beijing-Shanghai, Beijing-Guangzhou railway corridors--Tianjin-Baoding Intercity Railway(TBIR) as an example, analysis influence of railway construction on the spatial pattern of Jing-Jin-Ji Urban Agglomeration. Considering defects in the original analysis model, in the design of model analysis, will also increase the setting of parameters of the non road grid analysis, to further improve the accessibility analysis model, makes the analysis results more in line with the actual situation.

\section{Data sources and methods}

\subsection{Regional and transport network selection}

The study area for the Jing-Jin-Ji urban agglomeration, including Beijing, Tianjin two municipalities, and Shijiazhuang, Tangshan, Qinhuangdao, Langfang, Baoding, Zhangjiakou, Chengde city of Hebei Province (Figure 1). Within this regional context, it focused on more than 30 million urban population, the total 2013 GDP of about 4.8 trillion yuan, the region along the line connecting the city surrounded by approximately $60000 \mathrm{~km}^{2}$. As of 2016, the region's high-speed 
rail network density reached $1.071 \mathrm{~km} / 100 \mathrm{~km}^{2}$, second only to Pearl River Delta and Yangtze River Delta in the country. According to the design, the TBIR design speed of $250 \mathrm{~km}$, using mixed passenger and freight lines, and set aside cape passenger and freight lines. As a cross regional connection corridor, for the Northeast, Midwestern regions of the role of the connection as the impact of the post policy. The research emphasis on the analysis of the impact of the railway of the core functions of the district of Jing-Jin-Ji urban agglomerations, focus on the TBIR network influence on the regional accessibility and other traffic network yet considered.

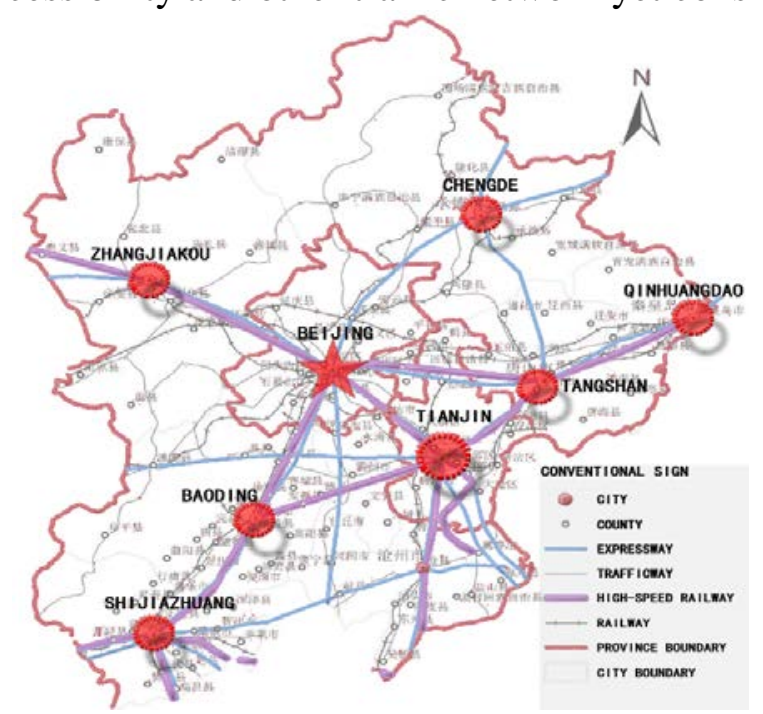

Fig.1 City’s Transport Network in Jing-Jin-Ji Urban Agglomerations

\subsection{Analysis method}

Based on Desktop10 ArcGIS software platform, through the study of the regional grid processing and analysis, we can get the regional time and cost of the results of the reach-ability analysis.

First of all, we needed to grid of research regions, classified of spatial distribution assigned to the space, and established a speed grid map. The analysis of railway traffic, with reference to the "Technical Standard of Highway Engineering" (JTGB01-2014) and the provisions of the national railway regulations and recommendations of the relevant documents, hourly railway 100km, high-speed railway $220 \mathrm{~km}$. Taking into account the scope of the study area, the grid is defined as $100 \mathrm{~m} \mathrm{x} 100 \mathrm{~m}$, and assuming that each grid is the same as that of the grid. According to the actual size of the grid, the speed of the raster map is converted to a time grid map. At last, the target point is calculated, and the time range map of the target point is obtained by using the software cost distance analysis module to calculate the time grid map and the space position of the target point.

Tab. 1 The Speed Evaluation of Grids with Different Attributes

\begin{tabular}{|c|c|c|c|}
\hline Transportation-Network Grids & Speed $(\mathrm{km} / \mathrm{h})$ & Non-Transportation-Network Grids & Speed(km/h) \\
\hline High-Speed Railway & 250 & Urban Residential & 30 \\
\hline Intercity Railway & 200 & Farmland & 15 \\
\hline Normal-Speed Railway & 100 & Meadowland & 20 \\
\hline Expressway & 110 & Woodland & 2 \\
\hline Trafficway & 80 & Rivers, Reservoirs and Other Water & 5 \\
\hline
\end{tabular}

Tab. 2 The Speed Evaluation of Grids with Different Elevations and Slopes

\begin{tabular}{|c|c|c|}
\hline Altitude(m) & Slopes (degree) & Speed $(\mathrm{km} / \mathrm{h})$ \\
\hline$<500$ & $0-15$ & According to the Land Use Type Assignment \\
\hline & $15-25$ & 1.0 \\
\hline & $>25$ & 0.5 \\
\hline 500 & $0-15$ & According to the Land Use Type Assignment \\
\hline & $>15$ & 0.5 \\
\hline
\end{tabular}

\section{Time accessibility analysis}

From the TBIR opened to traffic before and after the Tianjin area of accessibility analysis results of view. Before the opening of the railway (Figure 2a), 1.5h circle the main scope of distribution in 
East Hebei area, mainly is mainly administrative area of Tangshan, Qinhuangdao, and Baoding and Central South Hebei area is due to the inconvenience of traffic regions with poor accessibility. Among them, the area of $1 \mathrm{~h}$ rings, $1.5 \mathrm{~h}$ rings and $2.0 \mathrm{~h}$ rings accounted for $92.99 \%, 51.24 \%$ and $9.66 \%$ of the total area respectively, which accounted for $7.01 \%$ of the total area of $2 \mathrm{~h}$. After the opening of the railway (Figure 2b), The accessibility of Baoding and Central South Hebei area was significantly increased, and more areas were in the range of $1.5 \mathrm{~h}$. Traffic conditions, the area of $1.0 \mathrm{~h}, 1.5 \mathrm{~h}$ and $2 \mathrm{~h}$ rings increased to $9.66 \%, 55.83 \%$ and $95.66 \%$, and the area of the $2 \mathrm{~h}$ cycle was reduced to $4.34 \%$. Where time to reach the maximum change in the area of $1.5 \mathrm{~h}$, the area increased by $4.59 \%$.

From the time accessibility changes in space distribution and time accessibility of Jing-Jin-Ji urban agglomerations Eastern and northern area did not change, the area accounted for $81.1 \%$ of the total land area and time change of accessibility of the main areas of distribution in Baoding and Central South Hebei area. Baoding, Shijiazhuang are a substantial increase in the accessibility of areas, and presented a trend that the closer the distance TBIR is, the greater the accessibility change. Baoding is the largest area of accessibility change. Time changes in the area of less than 0.5h accounted for $9.51 \%$, 1 h changes in the area of $18.90 \%$ of the total area.

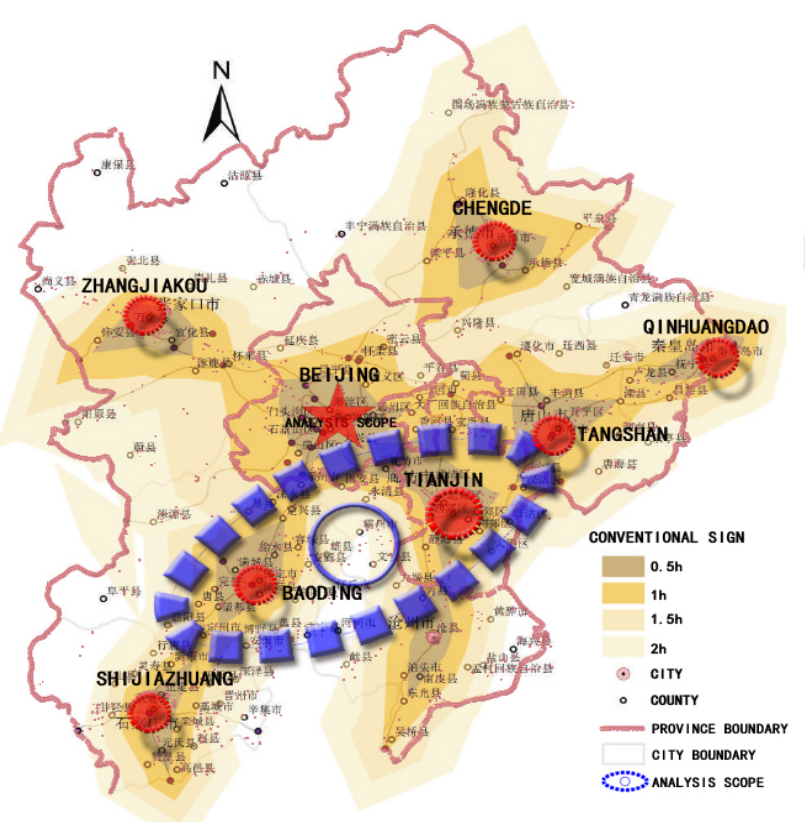

a. Before the Opening of TBIR

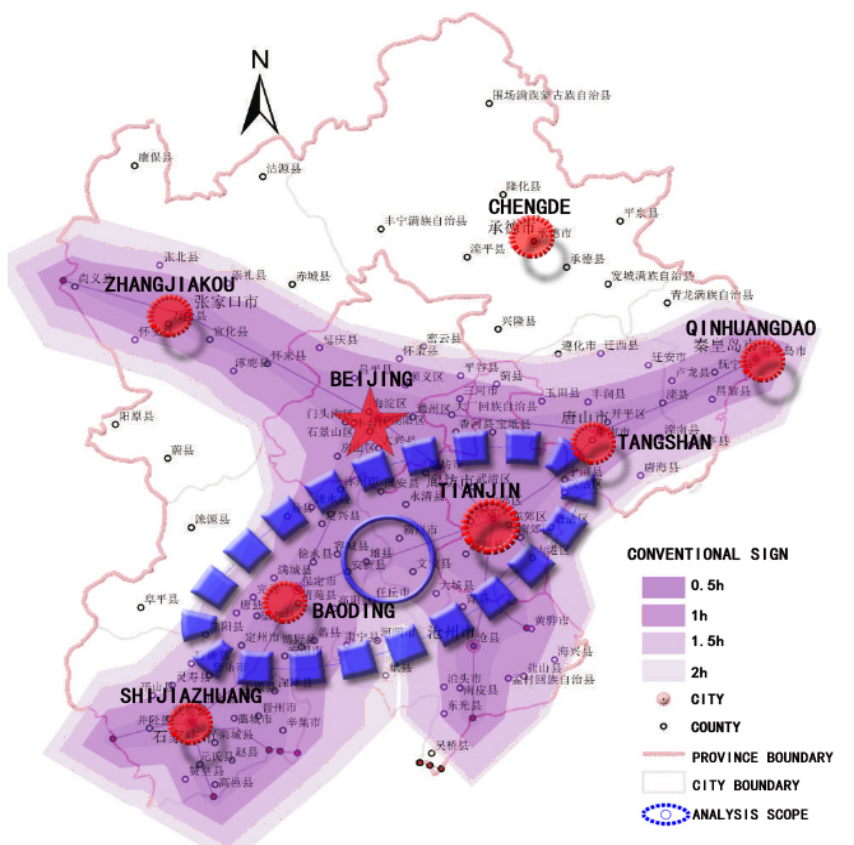

b. After the Opening of TBIR

Fig.2 The Regional Accessibility Before and After the Opening of TBIR

\section{Impact Analysis of TBIR}

\subsection{Promote the Coordinated Development of Regional Economy}

TBIR can enhance the location advantages of Tianjin and Baoding. Due to the lack of traffic channel, the radiation capacity of Tianjin to Central South Hebei area is obviously insufficient. At the same time, the economic development of Baoding and central and South Africa is lagging behind, the economic strength of Shijiazhuang has a big gap between Tianjin, so that the two ends of the development of the TIBR seriously unbalanced. The opening of TBIR cut space barrier, the railway of time-space compression effect made Baoding and Shijiazhuang traffic convenience greatly enhance between Tianjin, enhanced the location advantages of Central South Hebei, and the advantages of location accelerated economic development in Baoding area, notably in Baiyangdian New District will become even more prominent its position and policy advantages, Baoding area relatively rich in land resources will also be reflected the enormous advantage in resources, The opening of TBIR was conducive to reducing regional economic disparities, promoted Beijing-Tianjin-Hebei Collaborative Development. 


\subsection{Impact of Regional Traffic Pattern}

Jing-Jin-Ji urban agglomerations has formed a " (Beijing, Tianjin)-Tangshan-Qinhuangdao " and " (Beijing, Tianjin)-Baoding-Shijiazhuang " two traffic lines, it initially formed a diamond pattern of traffic space. The past 10 years, with the great development of the national railway construction, Beijing as a hub, has been in operation supersaturated state, unable to meet the future joint Beijing-Tianjin-Hebei Collaborative Development rapid growth in demand for transport. According to the feasibility study of TIBR, the opening of TBIR, will directly enhance the status as a hub of Tianjin, divert Beijing hub from East Hebei area and Northeast to Midwest railway transportation, meet the growing cross-boundary traffic demand. it has become an important channel of the core functions of the district of Jing-Jin-Ji urban agglomerations. Baoding will assume the functions of the transport hub, enhance Tianjin and East Hebei area transport links. With the construction of Beijing-Shijiazhuang intercity railway, Baoding-Xinzhou intercity railway, Baoding-Gu'an intercity railway, Tianjin-Shijiazhuang expressway and other transport corridors, the role in Jing-Jin-Ji urban agglomerations the region will also be increasingly sophisticated, with the potential to become even radiation south center city a major transportation hub in the Midwest. Meanwhile Shijiazhuang not only to increase contacts with Tianjin, but also further enhance the links East Hebei area, into the two hours traffic circle.

\subsection{Impact on regional industrial structure}

Factor flow is the way to optimize the allocation of resources, the lower the flow cost, the stronger the effect of factors on regional economic development. The opening of TBIR makes Jing-Jin-Ji urban agglomerations agglomeration resistance weakening, for different industries, the regional economic and industrial development to generate new patterns and differentiation, is conducive to regional industrial division of labor, promote regional industrial restructuring and upgrading and coordinated development. Based on polarization theory of economies of scale, Tianjin as an important trade, service center area, TBIR makes external radiation of Tianjin from the past to the northeast, further extends off the west direction, people, logistics and information flow of Central South Hebei will flow to Tianjin, Tianjin advantages in commerce, and services will be further strengthened, thereby strengthening the region "core-periphery" of the spatial structure. Tianjin will also generate the spillover effect of the economy, according to " theory of the distance elasticity estimates ", the distance between the city of Jing-Jin-Ji urban agglomerations of 1\% per, the amount of investment in the manufacturing industry, services, respectively, increased by $0.12 \% \sim 0.17 \%$. Preliminary estimates, the opening of TIBR, Central South Hebei cities can increase the $300 \sim 500$ billion yuan of GDP.

Based on the theory of complementary development of the professional division of labor, regional elements will optimize their combination according to their own conditions, to maximize the efficiency of resources and factors, and promote the common development of the industry. Abundant tourism resources can bring huge flow of people in Baoding area. Further promote the development and prosperity of accommodation, catering, shopping and other related industries. At the same time, due to the difference of Baoding, Central South Hebei area and Tianjin in land price, may also form similar to foreign "Bedroom Community" mode, namely the work in Tianjin, and living in the Baoding area work and lifestyle.

\subsection{Impact on regional logistics network}

Transportation logistics system is the link between the production, circulation, distribution and consumption of regional economic activities and the effective link between various departments and regions. The opening of TBIR, the direct effect is a significant speed of the transport logistics system. It makes East Hebei area and Central South Hebei area transportation do not bypass Beijing, becomes direct and rapid. For the container transportation in Shijiazhuang, TIBR makes the physical distance to the container port of Tianjin is greatly shortened, based on container in the distance near Huanghua port, it can also directly through the Tianjin exports to foreign, so the construction of the railway will caused by increased competition between the ports of Bohai Bay. Tianjin as an international shipping center, the port of high frequency, wide scope of shipping service advantage will become even more prominent, and Tianjin connection convenience increases 
will greatly increase the port hinterland, so as to promote the Central South Hebei area and Centerwest region economy development, also further enhance the functions of Tianjin as an international shipping center.

\section{Conclusion}

(1)TIBR significantly enhance the access time in Baoding, Central South Hebei area and Tianjin, $18.9 \%$ of the regional time accessibility has been improved, the time of up to change is the largest region of the original $1-2 \mathrm{~h}$ area. This area increase $4.59 \%$. Tianjin and Baoding are the main areas of change. (2) The "time-space compression" effect of TIBR has promoted the regional advantages of Baoding, and promoted the coordinated development of regional economy. (3) As the core functions of the district of Jing-Jin-Ji urban agglomerations important railway corridor, TIBR meet the increasing needs of passenger and freight transport in Baoding, Central South Hebei area and Tianjin, enhance the Baoding role as a transportation hub, thus change the traffic pattern. (4) TIBR will have different effects on different industries, the status of Tianjin's trade and service industry will be improved, and tourism and real estate will also benefit the Baoding area.

\section{References}

[1] JIN Fengjun, WANG Jiao"e, Railway Network Expansion and Spatial Accessibility Analysis in China: 1906 2000[J]. Acta Geographica Sinica. 2004, 59 (2):293-302.

[2] Lu Dadao, Function orientation and coordinating development of subregions within the Jing-Jin-Ji Urban Agglomeration[J]. Progress in Geography. 2015, 34(3):265-270.

[3] The 3rd Railway Survey \& Design Institute. Special report on preliminary design economic traffic volume from Tianjin to Baoding Railway[R]. 2009.17-24

[4] Li Pinghua, Lu Yuqi. Review and prospectation of accessibility research[J]. Progress in Geography. 2005, 24(3): 69-77.

[5] Sun Jianbo. Jinbao forecasting methods and apply on the intercity passenger traffic[D]. Dalian Jiaotong University. 2014 